The Be Phenomenon in Early-Type Stars, IAU Colloquium 175

ASP Conference Series, Vol. 214, 2000

M. A. Smith, H. F. Henrichs, and J. Fabregat, eds.

\title{
Summary and Future Directions
}

\section{J. M. Marlborough}

\author{
Department of Physics and Astronomy, The University of Western \\ Ontario, London, Ontario, Canada, N6A $3 K 7$
}

\begin{abstract}
A summary of the Alicante meeting, entitled The Be Phenomenon in Early Type Stars, is presented here. The emphasis at this meeting was on Be stars, but possible connections to other types of stars were also considered. Some suggestions for the directions of future approaches are provided. The meeting was dedicated to the late Mercedes Jaschek, Carlos Jaschek, and Arne Slettebak, all of whom made significant contributions to our current understanding of Be stars.
\end{abstract}

\section{Introduction}

By the end of a busy week in the sunny city of Alicante on the Spanish coast of the Mediterranean, the approximately 130 participants at IAU Colloquium 175 had listened to 5 papers which provided an overview of the Be phenomenon and the possible connections to other types of hot stars, such as $\mathrm{B}[\mathrm{e}]$ stars, and $\mathrm{B}$ and A supergiants, 6 papers outlining results from the use of new missions and technologies, 7 papers concerned with the production of variability, both periodic and aperiodic, in the atmospheres of isolated Be stars, 7 papers describing the circumstellar environment of Be stars, 2 papers discussing models of circumstellar discs, and 8 papers concerning the relationship of binary systems to the Be phenomenon. Six sessions, each fifteen minutes in length, were devoted to a review of the approximately 107 poster papers. In what follows I attempt to provide a summary of the conference and to propose some directions for future work which may increase our understanding of this type of star. I apologize in advance for omissions, misinterpretations, and neglect of material deemed to be very important by many of those present. The time for writing this review is relatively brief and the space available is finite. The material around Be stars is extended, rotating, and frequently variable on a variety of time scales. A wide range in density of the circumstellar matter is inferred from various diagnostics. Significant departures from LTE are expected to occur; simple analyses of data may therefore be highly misleading.

\section{Overview of the Be Phenomenon}

Balona presented an overview of the Be phenomenon. Interferometric studies of seven Be stars have shown that the $\mathrm{H} \alpha$ emitting region is of order ten stellar radii in extent and that the discs are very thin in a direction perpendicular to the equatorial plane. The $\mathrm{V} / \mathrm{R}$ variation can be understood in terms of a one 
arm, precessing density wave as described by Okazaki (1991) and Hummel and Hanuschik (1997). The maximum rotation speeds seem to cluster around $70 \%$ of the critical value. Two central problems remain: what drives the mass loss and what produces time variability? Episodic mass loss is common in Be stars; it may be connected to photospheric activity, which may be magnetic in origin. Variability may have more than one origin; non-radial pulsation or material trapped in localized magnetic loops are two possibilities. The differences between $\mathrm{B}$ and $\mathrm{Be}$ stars may be connected with the presence of both rapid rotation and magnetic fields.

Harmanec discussed the basic physical properties of Be stars. Determination of these is difficult because of a lack of detailed knowledge of the variability of each star. For example, problems with $v \sin i$ values still exist; for $\gamma$ Cas these range from $\sim 200$ to $500 \mathrm{~km} \mathrm{~s}^{-1}$. To quote from Slettebak (1987), "Little direct information about the fundamental parameters of the underlying Be stars is presently available, and that information which can be obtained is not always easy to interpret because of envelope effects and the shape distortion and nonisotropic radiation fields which are probably characteristic of rapidly-rotating stars."

Zickgraf considered the $\mathrm{Be}, \mathrm{B}[\mathrm{e}]$ connection. He emphasized that the $\mathrm{B}[\mathrm{e}]$ designation defines an environment rather than simply a type of object; the $\mathrm{B}[\mathrm{e}]$ stars therefore do not necessarily form a homogeneous group. There might, however, be a connection between the lower luminosity $\mathrm{B}[\mathrm{e}]$ stars and Be stars. Kaufer compared Be stars to B supergiants; Verdugo compared Be stars to A supergiants. $\mathrm{H} \alpha$ emission is generally present in both types, but can vary on long time scales. The supergiant winds show both radial and azimuthal structure similar to Be stars. Rotational modulation of spectroscopic features occurs in both types, suggesting perhaps some surface structure origin.

\section{New Missions and Technologies}

Briot reviewed the Hipparcos data for the sample of more than 500 Be stars. On average Be stars are brighter than normal B Stars, but the magnitude difference depends on spectral type. Interferometric study of Be star discs was discussed by Stee. Milliarcsecond spatial resolution can now be used to study the envelopes of Be stars. Solid body rotation can be ruled out, but the current data do not distinguish between Keplerian rotation and angular momentum conservation. For $\psi$ Per, the same position angle of the disc is obtained from both optical and millimeter data. Linfield described the ST3 mission, a visible wavelength interferometer which will employ two spacecraft in heliocentric orbit and provide baselines of $40-200 \mathrm{~m}$; it is to be launched in 2003. The instrument is expected to be capable of resolving Be discs both spatially and in velocity.

Waters reviewed the properties of Be stars inferred from data at infrared and radio wavelengths. At these long wavelengths the spectrum of a $\mathrm{Be}$ star is dominated by hydrogen emission from the circumstellar matter, the emission coming from recombination lines and free-bound and free-free processes. For $\gamma$ Cas the series limits of the Humphreys and Hansen-Strong series are detected, as well as those corresponding to series whose lower levels have principal quantum numbers 8,9 , and 10 . A few He I lines are also observed. The hydrogen line 
strengths do not follow case B recombination line theory; the line strengths and widths suggest many are optically thick.

Cohen discussed x-ray emission from isolated Be stars. In OB stars an extended plasma with temperature of order a few million degrees exists. This hot gas is thought to be associated with shocks in the wind, the shocks arising from the line force instability. Significant variability does not occur. Be stars might be expected to be different compared to B stars because Be stars have higher mass loss rates, show deviations from spherical symmetry yielding higher electron number densities, and may have magnetic fields and hence enhanced $\mathrm{x}$-ray emission. ROSAT data show a steep fall off in $\mathrm{x}$-ray detection for normal $\mathrm{B}$ stars of luminosity class $\mathrm{V}$ as the stellar temperature decreases. However this steep decline is not seen in a sample of $7 \mathrm{Be}$ stars ranging in spectral type from B1.5 to B7. Perhaps rapid rotation, disks, and/or pulsation somehow enhance $\mathrm{x}$-ray emission in Be stars.

\section{Time Variability - The Role of Stellar and Atmospheric Processes in Isolated Be Stars}

Baade described periodic phenomena observed in Be stars. One might divide these into two types, photospheric and extra-photospheric or exo-photospheric. Nonradial pulsations (NRP) are detected in Be stars. The rapid V/R variations and variations of the Balmer discontinuity arise from NRP induced temperature variations. Beating of different modes is likely to be an ingredient in mass loss. Later type Be stars may be different, however, because the amplitude/incidence of NRP decreases with decreasing effective temperature.

Aerts reviewed multiple mode detections using data obtained from surveys, both photometric and spectroscopic. Photometric surveys of 17 stars at ESO and 22 at SAAO revealed no multiperiodicity and, perhaps more interestingly, no variation for stars cooler than B7. In the Hipparcos data, Hubert and Floquet identified 13 candidates as potentially being multiperiodic. In another survey containing stars from both Balona and Hubert and Floquet, 11 stars of a sample of 50 had more than one period. However it is not presently obvious that any of these multiperiods is stable. Data sets obtained over a longer time scale may be necessary to resolve this issue.

Henrichs described the $\mathrm{MuSiCoS}$ (multi site, continuous network) campaign. One result is new strong evidence for a varying magnetic field in the star $\beta$ Cep. The magnitude of the field is about 100 gauss. The star was presumably a more rapid rotator in the past and has been spun down. However, does $\beta$ Cep satisfy the definition of a Be star? Stefl outlined the Heros campaign. Heros is a spectrograph with a high resolving power and a wavelength range of 3450-8620 $\AA$. Outbursts of several Be stars have been followed. Hao reviewed the Asian contribution to campaigns devoted to a variety of Be stars.

Peters discussed the results from IUE observations. Approximately 18 stars were followed during the period 1985-1996. In $28 \mathrm{Cyg}$, bumps travelling across the profile in time suggest NRP. Larger variations seen at shorter wavelength may imply temperature variations. If Kurucz models are used, a hemisphere averaged, temperature variation of about $3000^{\circ} \mathrm{K}$, is needed to fit C III $\lambda 1247$. The flux of $\alpha$ Eri varies by $30 \%$ in the far UV on a time scale of 1.28 days; the IUE flux variation at $\lambda 1450 \AA$ shows a similar period. 
Smith described a range of evidence for activity, i.e. aperiodic or nonrepetitive variability, in classical Be stars. Rapid changes in the strength of emission lines, e.g. H $\alpha$, suggest ionization changes due to local heating. High velocity absorption components are seen on the short wavelength side of some absorption lines in $\mu$ Cen and $\lambda$ Eri, perhaps the result of some localized ejection process. Other examples also occur. These aperiodic events are common in $\mathrm{Be}$ stars, independent of whether the star shows evidence of NRP. Is the ultimate source for the energy of these localized events magnetic in origin? If Smith's suggestions regarding magnetic activity are correct, it is important to confirm directly the presence of magnetic fields in the atmospheres of Be stars.

Mathys reviewed attempts to detect directly magnetic fields in Be stars. In Babcock's 1958 catalogue, there are at best two doubtful detections among the 12 $\mathrm{Oe}, \mathrm{Be}$ stars. New data for $\lambda$ Eri yield an upper limit of $0 \pm 6 \mathrm{kG}$; however fields of $3 \mathrm{kG}$ could be present but not detected with the technique used. Haisch outlined the physical requirements for flares in stars. Stellar analogues of solar flares are well known. The basic requirement for a flare is a current sheet trapped between oppositely directed magnetic fields. To obtain this one needs differential rotation and convection in addition to the magnetic field. How do such conditions occur in stars with convective cores and radiative envelopes? Airapetian noted that a poloidal magnetic field can be produced in the convective core of a massive star, but it would require a long time to rise to the surface. However the interaction of global oscillations, which exist in early type stars with rapid rotation, can lead to Rossby waves, which are incompresssible, non-axisymmetric oscillations, that can introduce helicity and produce a magnetic field. Cassinelli and MacGregor considered the rise of a magnetic flux tube through the radiative envelope of a hot star. The tube will rise but eventually stalls when a balance between magnetic tension and Coriolis forces occurs. Winds and/or circulation currents may allow the field to reach the surface.

\section{The Circumstellar Environment of Be Stars}

$\mathrm{K}$. Bjorkman reviewed the role of polarimetry as a diagnostic of the geometrical and physical conditions in the circumstellar envelopes of hot stars in general and of Be stars in particular. Recently, improved polarimeters and spectropolarimeters have become available. Compared to earlier instruments these provide better time coverage, improved $\mathrm{S} / \mathrm{N}$ ratio, and allow extensions to the $\mathrm{UV}$ and IR spectral regions. More stars have been investigated using both improved physics and better modelling. Low polarization is found in the near UV due to line blanketing by disc material. Large supression of polarization in A stars also occurs; this agrees with the conclusions reported by Clayton and Marlborough and Dominy and Smith approximately twenty years ago.

Hummel described line profile modelling of Be star discs. Shell line profiles are consistent with Keplerian rotation and the lack of radial motion in the disc. Intercomparison of data from the UV and optical suggest the UV wind and the disc evolve independently. Numerical modelling, which incorporates NLTE radiative transfer in three dimensions, shows that global disc oscillations can account reasonably well for both the observed profiles of $\mathrm{H} \alpha$ and their long term variability. Discs larger than 5 stellar radii seem to be needed to obtain good 
agreement between predicted line profiles and observations, especially for small inclinations.

Telting discussed variability in discs. In a few cases disc growth rates have been determined. For Pleione, Hirata (1995) used shell lines and obtained a growth rate of $6 \times 10^{-11} \mathrm{M}_{\odot} \mathrm{y}^{-1}$. For $\mu$ Cen, Hubert and Floquet(1998) and Rivinius (1998) obtained a growth rate of $2 \times 10^{-9} \mathrm{M}_{\odot} \mathrm{y}^{-1}$. Insofar as the $\mathrm{V} / \mathrm{R}$ variations are concerned global, one-armed oscillations seem to be the only possible explanation. These variations are cyclic on time scales of 2-13 years. In $66 \mathrm{Oph}$, Hanuschik et al (1995) detected the onset of $V / R$ variations during a time when $\mathrm{H} \alpha$ was increasing in strength. Does this mean that a critical disc mass is needed before V/R variability can occur? Spectacular variations occur in some stars, e.g. $59 \mathrm{Cyg}$ and $\gamma$ Cas. The $\mathrm{V} / \mathrm{R}$ cycle is not correlated with the orbital period of any companion star; thus disc growth is due to the $\mathrm{Be}$ star itself. However companions do affect the disc in some cases, e.g. X Per, A0535+26.

Ignace considered polarization in non-axisymmetric discs. One would expect periodic polarimetric variations if non-axisymmetric structures exist, the source of the $V / R$ variation being an obvious candidate. If the disc were optically thin and unresolved no net change in polarization would be expected. However, multiple scattering and/or disc emission effects might lead to disc selfpolarization. Time variable polarization might arise from any non-axisymmetric structure.

Oudmaijer discussed $\mathrm{H} \alpha$ spectropolarimetry of $\mathrm{B}[\mathrm{e}]$ and Herbig Be stars. Several Herbig Be stars show changes in polarization across $\mathrm{H} \alpha$, thus confirming the presence of discs in these objects. $\mathrm{B}[\mathrm{e}]$ stars show large polarization changes across $\mathrm{H} \alpha$, supporting the view that such objects have dense equatorial discs.

Okazaki reviewed the long term $V / R$ variations and their explanation in terms of global, low frequency oscillations in near Keplerian discs. The eigenmodes with $m=1$ have properties consistent with the observed properties of the $\mathrm{V} / \mathrm{R}$ variations. However these eigenmodes with $m=1$ are present only in Keplerian discs and mode confinement only occurs for subsonic radial speeds. Hence this explanation requires that $\mathrm{Be}$ discs be viscous excretion discs.

J. Bjorkman described the formation and structure of circumstellar discs. Some $20-30 \%$ of Be stars may have arisen by accretion during the pre main sequence phase of evolution and/or by mass transfer in a binary system. The remaining $70-80 \%$ must generate discs by outflow. The wind compressed disc is one possibility, but suffers from some problems. A crucial problem arises from the generally low disc density; the matter in the wind compressed disc does not have enough angular momentum to form a disc of sufficient density to account for the observed infrared excesses. Independent of the model for the disc, the process which transfers matter from the star to the disc is still unknown. Alternatively, some discs may be Keplerian. However the angular momentum transfer process needed to produce a Keplerian disc is also unknown. Millar outlined how the ionization-excitation equilibrium and the kinetic temperature can be obtained simultaneously and self-consistently as a function of position in the circumstellar matter around a Be star.

Hanuschik discussed the various types of Fe II shell lines and how these lines can be used as probes of the circumstellar disc. There are, in general, three 
types of shell lines: broad, symmetric lines with width of order $2 v \sin i$; narrow symmetric lines with width $<10 \mathrm{~km} \mathrm{~s}^{-1}$; and broad, asymmetric lines whose shape varies cyclically on a time scale of years. The properties of the first two groups suggest they are formed in Keplerian discs; the variations of the third group presumably arise because of the one arm density wave.

Coe reviewed Be stars in X-ray binary systems. There are two types of such systems; one contains a supergiant B star as the primary and the other contains a Be star; the companion in all cases is thought to be a degenerate star, either a neutron star or a black hole. This type of system is quite common; many are known in both the Galaxy and the Magellanic Clouds. White dwarf companions seem to be less common, but this might be a selection effect. The $\mathrm{x}-$ ray characteristics are more or less steady in systems containing a $B$ supergiant, whereas flares are more common in systems with Be stars. Perhaps mass transfer from the Be star is more episodic.

It seems unlikely that all $\mathrm{Be}$ stars arise in mass transfer binaries as proposed by Kriz and Harmanec (1975). However some Be stars may be post mass transfer binaries with the companion being a helium star; $\phi$ Per seems to be a good candidate, and Gies outlined the evidence supporting this suggestion.

Owocki discussed the current status of the wind compressed disc model. In a rapidly rotating star with a radially driven stellar wind, a pole equator asymmetry arises naturally; conservation of angular momentum of the matter ejected from the star leads to an equatorial, wind compressed disc (WCD) being formed. However, if the line force is treated using the Sobolev approximation, the non-radial components of the line force prevent a WCD being formed. But real stellar winds have significant small scale structure due to the line driving instability. Owocki discussed some preliminary results based on attempts to include the non-local character of the line force. No strong poleward flow occurs, but also no WCD.

Petrentz described two dimensional models for radiation driven winds and applied these to $\mathrm{B}[\mathrm{e}]$ stars. In the context of a CAK wind, the radiation force parameters $\mathrm{k}, \alpha$, and $\delta$, are permitted to vary with position. Fe III is a major contributor to the line force. At a sufficiently large mass loss rate, a switch from Fe III to Fe II occurs, leading to less wind driving and a denser wind, analogous perhaps to the bistability mechanism of Lamers and Pauldrach (1991).

\section{Future Directions}

It has been common to use the term Be phenomenon to describe the subject of interest at this conference. However this term may be too narrow in scope, if interpreted literally, because many of the properties used to define Be stars are also common to or demonstrated by some $\mathrm{O}$ stars and $\mathrm{A}$ stars. Hence I suggest that the term OBA phenomemon, or something similar, may be a more appropriate descriptive term with which to characterize the stars of interest. Among O stars, $\zeta$ Oph (O9.5V, vsini $\left.=396 \mathrm{~km} \mathrm{~s}^{-1}\right)$ and HD $60848(\mathrm{O} 7-8, v \sin i$ $=362 \mathrm{~km} \mathrm{~s}^{-1}$ ) satisfy all the criteria used to define a Be star.

Some A stars show many of the properties of Be stars. However, $\mathrm{H} \alpha$ emission occurs generally only in the hottest A stars; an example is the A type shell star 1 Del (A0V or A1e shell, vsini $=320 \mathrm{~km} \mathrm{~s}^{-1}$ ). Dominy and Smith (1977) 
studied a number of A type shell stars, cooler than spectral type A5, and concluded that the shells are essentially neutral. Clayton and Marlborough (1980) came to the same conclusion from their failure to detect continuum linear polarization in a sample of eight A type shell stars, two of which were hotter than spectral type A5. The coolest star displaying the OBA phenomenon may be 14 Comae (A9-F0 III, $v \sin i=227 \mathrm{~km} \mathrm{~s}^{-1}$ ). Jaschek et al. (1986) found that of a sample of $19 \mathrm{Ae} / \mathrm{A}$ shell stars observed by IRAS, only 8 were found to have an infrared emission component attributable to dust. The additional infrared emission from the remaining objects presumably arises from circumstellar gas with a low degree of ionization. Abt et al. (1997) have noted that many rapidly rotating A stars of luminosity class V show shell lines of Ti II, which appear and disappear on time scales of decades. These lines are seen only in stars with $v \sin i>175 \mathrm{~km} \mathrm{~s}^{-1}$, suggesting they are formed in discs seen in absorption only at large inclination angles. Thus there are some rapidly rotating $A$ type stars which show evidence for the presence of equatorially concentrated circumstellar matter, which is largely neutral. These may be the A star analogues of Be stars. The internal structure of massive main sequence stars consists of a convective core and a radiative envelope. The internal structure reverses for $\mathrm{L} \lesssim 10 \mathrm{~L}_{\odot}$ and $\mathrm{T}_{\text {eff }}<7500 \mathrm{~K}$. If a convective core/radiative envelope is required for a Be star, one would not expect to find Be-like activity in stars near the main sequence and cooler than $7500 \mathrm{~K}$.

The upper portion of the HR diagram is populated by a variety of types of stars, such as LBVs, WR stars, B[e] stars, etc. The B[e] stars are of particular interest because there exists evidence which seems to suggest a connection to Be stars. As a group, $\mathrm{B}[\mathrm{e}]$ show an absorption line spectrum characteristic of a B star, recombination emission lines of $\mathrm{H} \mathrm{I}, \mathrm{He} \mathrm{I}$, narrow permitted emission lines of $\mathrm{Fe} \mathrm{II}, \mathrm{Mg} \mathrm{II}, \ldots$, forbidden emission lines of [Fe II], [S II], ..., a strong infrared excess due to hot dust, broad UV stellar wind lines for some, a large range in stellar luminosity but no indication of variability, and continuum linear polarization suggesting a disc structure for some of the circumstellar material. Gummersbach et al (1995) have shown that the B[e] phenomenon in the Magellanic Clouds extends to stars whose inferred main sequence masses lie in the range $7-15 \mathrm{M}_{\odot}$, the same mass range for Be stars. In a poster paper Miroshnichenko and K. Bjorkman discussed a sample of Be stars with far IR excesses from IRAS data. They concluded that the IR excesses are more likely due to circumstellar dust. Might Be stars of this type and the 8 stars of Jaschek et al, with IR emission attributable to dust, be transition objects connecting classical Be stars with $\mathrm{B}[\mathrm{e}]$ stars?

The strength of emission lines of Be stars frequently varies with time; sometimes emission lines disappear completely, sometimes they appear in a star which previously had only an absorption line spectrum. However, there are some $\mathrm{Be}$ stars, the so-called stable shell stars, which show no changes of velocities of shell lines or spectra over periods of order fifty years (Gulliver 1981 and references therein). An example is HD 193182 (B7-B8), perhaps the best example of a stable shell star. Hydrogen absorption lines can be seen to $\mathrm{H} 42$ or H43 (a reproduction of the spectrum is shown in Physics Today volume 2, April, 1949). No changes in the spectrum have been seen over a period of at least sixty years (Gulliver 1981). For three of these stars the total width of the Ca II K line at the continuum exceeds $2 v \sin i$. Why is variability apparently less common 
among the cooler $\mathrm{Be} / \mathrm{Ae}$ stars compared to the hotter ones? Is this simply a result of the decrease in the amplitude/incidence of NRP with decreasing effective temperature, as Baade suggested in his review? Or are the cooler Be/Ae stars fundamentally different from the Oe/Be stars?

There is a variety of evidence demonstrating similar anomalous line widths in other Be stars. Hanuschik (1987) found that total Fe II line width, at the base of the line, exceeded $2 v \sin i$ for Fe II emission lines. Waters and Marlborough (1994) noted that the estimated width of $\mathrm{Br} \gamma$ in UKIRT spectra of 59 Cyg and $\psi$ Per exceeded $2 v \sin i$ for each star. Hony et al. (2000) discovered that the weakest $\mathrm{H} \mathrm{I}$, IR lines in the ISO spectrum of $\gamma$ Cas have FWHMs of $\geq 550$ $\mathrm{km} \mathrm{s}{ }^{-1}$, whereas $\mathrm{v} \cdot \sin \mathrm{i}=230 \mathrm{~km} \mathrm{~s}^{-1}$. Several questions arise. Have $\mathrm{v} \cdot \sin \mathrm{i}$ values for Be stars been systematically underestimated? Do all Be stars rotate at the critical speed? Are the discs of Be stars excretion discs as Porter (1999) has recently suggested?

Improvements in the physics of circumstellar discs are ongoing. Millar and Marlborough (1999) have shown how the kinetic temperature of the gas in the circumstellar matter of a Be star can be predicted self consistently, and applied this technique to both a hot and a cooler Be star. Subsequent improvements include the addition of metals, iron, for example. More realistic model atoms, with better treatment of the envelope line radiation, are also being developed.

In the past few years stellar opacities have improved dramatically with the completion of the OPAL opacities, Iglesias and Rogers (1991), and the OP opacities, Seaton et al.(1994). Both sets of opacities, based on different environmental models, generally agree well with each other. In view of these advances it might now be appropriate to make a detailed investigation of the structure of the equatorial regions of rapidly rotating $B$ stars, taking into account, explicitly, both the larger equatorial radius due to the departure of the star from spherical symmetry and the local radiation force using the new opacities. Pamyatnykh (1998) described calculations of this type, which however did not include departures from spherical symmetry. Furthermore, again in view of the improved opacities, it may also be informative to apply the bi-stability investigation of Lamers and Pauldrach (1991) to the equatorial regions of Be stars, again including the increase in the equatorial radius due to rapid rotation.

Smith and Robinson (1999) have discussed the results of a multiwavelength campaign concentrating on $\gamma$ Cas. They conclude that magnetically generated structures on and/or above the stellar surface are responsible for the $\mathrm{x}$-ray flares detected. They also detect UV activity. Both x-ray and UV activity have the same period, which they interpret as the rotation period of the star. In a plane parallel model atmosphere at a wavelength of $5000 \AA$ for a star with effective temperature $25000 \mathrm{~K}$ and $\log g 4.0$, the He II, He III ionization zone occurs at optical depth $\sim 10$ and is convective. At lower temperatures the $\mathrm{He} \mathrm{I}, \mathrm{He} I I$ ionization zone shows the same effect at a somewhat smaller optical depth. If the star had a weak, global magnetic field, convection might lead to a disordered field in which magnetic reconnection could occur. The energy released in this manner might be the source of the $\mathrm{x}$-ray flares and the UV activity. Future investigations will determine whether or not these suggestions have any relevance first to $\gamma$ Cas and then perhaps to other Be stars. 
Acknowledgments. I thank John Landstreet for providing the details of the atmospheric models which reveal the existence of convective layers associated with the He II - He III, and the He I - He II ionization zones, and Carol Millar for her comments on this summary. I acknowledge financial support from the Natural Sciences and Engineering Research Council of Canada.

\section{References}

Abt, H. A., Tan, H., and Zhou, H. 1997, ApJ, 487, 365

Clayton, G. C., and Marlborough, J. M. 1980, ApJ, 242, 165

Dominy, J. F., and Smith, M. A. 1977, ApJ, 217, 494

Gulliver, A. F. 1981, ApJ, 248, 222

Gummersbach, C. A., Zickgraf, F.-J., and Wolf, B. 1995, A\&A, 302, 409

Hanuschuk, R. W. 1987, A\&A, 173, 299

Hanuschik, R.W., Hummel, W., Dietle, O., and Sutorius, E. 1995, A\&A, 300, 163

Hirata, R. 1995, PASJ, 47, 195

Hony, S., Waters, L. B. F. M., Zaal, P. A., de Koter, A., Marlborough, J. M., Millar, C. E., Trams, N. R., Morris, P. W, and de Graauw, Th. 2000, A\&A, 357,1013

Hubert, A. M., and Floquet, M. 1998, A\&A, 335, 565

Hummel, W., and Hanuschik, R. W. 1997, A\&A, 320, 852

Iglesias, C. A., and Rogers, F. J. 1991, ApJ, 371, 408

Jaschek, M., Jaschek, C., and Egret, D. 1986, A\&A, 158, 325

Kriz, S., and Harmanec, P. 1975, BAIC, 26, 65

Lamers, H. J. G. L. M., and Pauldrach, A. W. A. 1991, A\&A, 244, L5

Millar, C. E., and Marlborough, J. M. 1999, ApJ, 516, 276

Okazaki, A. T. 1991, PASJ, 43, 75

Pamyatnykh, A. 1998, in Variable and Non-spherical Stellar Winds in Luminous Hot Stars, eds. B. Wolf, O. Stahl, and A. W. Fullerton, Springer-Verlag, Berlin, p. 320

Porter, J. M. 1999, A\&A, 348, 512

Rivinius, Th., Baade, D., Stefl, S., Stahl, O., Wolf, B., and Kaufer, A. 1998, A\&A, 333, 125

Seaton, M. J., Yan, Yu, Mihalas, D., and Pradhan, A. K. 1994, MNRAS, 266, 805

Slettebak, A. 1987, in Physics of Be Stars, eds. A. Slettebak and T. P. Snow, Cambridge University Press, Cambridge, p. 24

Smith, M. A., and Robinson, R. D. 1999, ApJ, 517, 866

Smith, M. A., Robinson, R. D., and Corbet, R. H. D. 1998a, ApJ, 503, 877

Smith, M. A., Robinson, R. D., and Hatzes, A. P. 1998b, ApJ, 507, 945

Waters, L. B. F. M., and Marlborough, J. M. 1994, in Pulsation, Rotation and Mass Loss in Early-Type Stars, eds. L. A. Balona, H. F. Henrichs and J. M. Le Contel, Kluwer Academic Publishers, Dordrecht, p. 399 